\section{LOS PELIGROS DE LA SOBRESTIMULACIÓN EN LA METRÓPOLIS MODERNA: GEORG SIMMEL Y SU LECTURA DEL NUEVO URBANITA}

\author{
Sonsoles Hernández Barbosa \\ Universitat de les Illes Balears \\ ORCID iD: https://orcid.org/0000-0002-4277-0578 \\ sonsoles.hernandez@uib.es
}

Cómo citar este artículo/Citation: Hernández Barbosa, S. (2019). Los peligros de la sobrestimulación en la metrópolis moderna: Georg Simmel y su lectura del nuevo urbanita. Arbor, 195 (791): a497. https://doi.org/10.3989/arbor.2019.791n1010

Recibido: 16 febrero 2018. Aceptado: 4 junio 2018.

RESUMEN: A finales del siglo XIX, la economía capitalista y las nuevas formas de consumo que trae implícitas tratando de seducir al individuo urbano con multitud de estímulos suponen un cambio en el modo en que este experimenta la ciudad. Esto conlleva la aparición de nuevas patologías, como la neurastenia, producto de un supuesto exceso de demanda del cerebro. Estos efectos fueron abordados en la época por Georg Simmel, quien se interesó por la reacción que sobre el individuo produce la urbe capitalista ligada a la "intensificación de la vida nerviosa". En el presente artículo se indaga en la lectura que Simmel plantea de los efectos que la ciudad moderna ejerce en el ciudadano. Me centro particularmente en la cuestión de la saturación de estímulos para tratar de entender no solo cómo creía que la ciudad afectaba al ciudadano en la época sino también el novedoso papel que otorga a la sensibilidad como intermediaria del vínculo entre individuo y ciudad.

PALABRAS CLAVE: Georg Simmel; sensibilidad urbana moderna; sobrestimulación; vida mental en la modernidad; historia cultural de los sentidos.

\section{THREATS OF OVERSTIMULATION IN MODERN METROPOLIS: GEORGE SIMMEL'S APPROACH TO THE NEW CITY DWELLER}

Copyright: (C) 2019 CSIC. Este es un artículo de acceso abierto distribuido bajo los términos de la licencia de uso y distribución Creative Commons Reconocimiento 4.0 Internacional (CC BY 4.0).

ABSTRACT: At the end of the $19^{\text {th }}$ Century, the capitalist economy and new forms of consumption associated with it set out to seduce the city dweller with numerous stimuli that transformed their experience of the city. This involved the appearance of new pathologies like neurasthenia, related to a supposed excess cerebral demand. Sociologist Georg Simmel became interested in the effects of the capitalist metropolis on the individual linked to the "intensification of nervous life". In this paper I address Simmel's view of the city dweller's reaction to the novelties of modern city life. Special attention is paid to the question of overstimulation, approaching not only how the influence of urban life on the individual was understood, but also the new role given to sensitivity as an intermediary between the individual and the city.

KEYWORDS: Georg Simmel; modern urban sensibility; overstimulation; mental life in modernity; cultural sensory history. 
A finales del siglo XIX se produce una transformación en las ciudades europeas. Al tiempo que aumentan en densidad de población y dimensiones, su morfología experimenta importantes cambios. Es el caso de París y Londres que rivalizan por convertirse en capitales de la modernidad. En París, durante el Segundo Imperio, la reforma haussmanniana (1853-1868) se impone sobre la villa medieval, proceso de planificación y renovación urbana que será replicado en otras ciudades occidentales ${ }^{1}$. Callejones lóbregos quedan atravesados por grandes bulevares jalonados de verde y fachadas que dan un nuevo aspecto a la ciudad a la vez que responden a las medidas higienistas imperantes en la época, con nuevos alcantarillados y un mobiliario urbano específico. La aparición de la luz de gas también contribuye a esta renovación, que en el final de siglo se tornará luz eléctrica, impulsada sobre todo a partir de la exposición de la electricidad de 1881 (Bertrand, 1996, pp. 39-40). Estas transformaciones en la ciudad inciden no solo en su imagen física sino también en el paisaje sonoro ya que prácticas como la venta ambulante -con sus consignas específicas (cris)- tienden a ser reprimidas, tanto por la legislación como por la propia dinámica de las nuevas infraestructuras comerciales (Boutin, 2015)2.

Sin duda, la creciente economía capitalista tiene mucho que ver en este proceso, al hacer de las ciudades espacios de espectacularización a los que contribuyen las exposiciones universales y los grandes almacenes (Hahn, 2009) ${ }^{3}$. Estos espacios surgen de la evolución de los pasajes, de los que mantienen aspectos formales, como la iluminación cenital a través de vidrieras, y cuyo desarrollo aparece en paralelo a la arquitectura del hierro. Su desarrollo coincide con el auge de la economía capitalista a partir de 1848 y ejemplifican el intento de poner a disposición de la mayor cantidad de público, particularmente de la burguesía emergente, el máximo de productos (Marrey, 1979). Para que este consumo sea lo más efectivo posible se desarrollan estrategias que tratan de seducir a ese potencial consumidor burgués, captar su atención, en un contexto de competitividad que busca posicionar de manera privilegiada el producto de la marca propia frente al resto, lo cual se enmarca en una incipiente industria del marketing.

Las nuevas formas de consumo y la intensificación de estímulos que traen implícitas suponen un cambio en el modo en que se vive la ciudad. La experiencia citadina pasa a estar marcada por la novedad, por el sobrevenir constante de estímulos y acontecimientos, una fugacidad de impresiones que había apuntado Charles Baudelaire en su texto El pintor de la vida moderna (Le peintre de la vie moderne, 1863) como característica fundacional de la modernidad (Baudelaire, 1863/2000).

Georg Simmel (1858-1918) constituye una figura clave a la hora de entender las transformaciones que se estaban gestando en la ciudad capitalista, que concibe como el espacio representativo de la vida moderna. En este artículo veremos, por este orden, cómo Simmel aborda las nuevas condiciones que impone la urbe moderna, cómo considera que estas influyen en los comportamientos cotidianos de sus habitantes y cómo afectan a la psicología del individuo de este momento de entresiglos. Cruzaré para ello sus textos sobre la ciudad "Roma, Florencia, Venecia" (1898) y "Las grandes ciudades y la vida intelectual" (1903) con la lectura sociológica de la percepción sensorial que presenta en su "Digresión sobre la sociología de los sentidos" (1907) ${ }^{4}$. Me interesa la mirada "de cerca" de Simmel, propia de un perspicaz flâneur del Berlín de la época (Faria Peres, Flores Durán, Pacini de Medeiros y Alburquerque, 2011, pp. 95-97), capaz de poner sobre la mesa algunas de las transformaciones que se estaban produciendo en la ciudad y que a su vez apuntan a preocupaciones del momento, lo que ha conducido a su consideración como Herr Zeitgeist o encarnación del espíritu de su época (Cuesta, 2016, p. 15).

\section{DE LA VIDA PREMODERNA A LA VIDA EN LA GRAN URBE}

La ciudad constituye el entorno natural de los objetos de reflexión de Simmel: el dinero, las exposiciones universales, la moda, el coqueteo; objetos todos ellos integrados en el hilo conductor de la experiencia cotidiana moderna. La elección de estas temáticas se encuentra en consonancia con su particular sociología: una sociología fragmentaria, enfocada desde una óptica microscópica. Cada una de estas temáticas se encuadra en una constelación de elementos donde todos ellos se presentan relacionados respondiendo a una concepción de la sociedad no como algo acabado, fijo y estable sino como un flujo en constante cambio en el que la alteración de uno de los parámetros afecta necesariamente a los otros (Faria Peres et al., 2011, pp. 97-100). Su aproximación a la ciudad debe enmarcarse así en su Sociología (1908) como parte de un estudio de la interacción social; tengamos en cuenta que, como Frisby apunta, Simmel otorga particular importancia a la experiencia cotidiana y al conocimiento de los individuos como fundamentación de su sociología, aspectos ambos en los que me basaré para 
estudiar su lectura de la experiencia urbana moderna (Frisby, 2008, p. 23) ${ }^{5}$.

Para entender la interpretación que Simmel hace de la gran ciudad hay que referirse, en primer lugar, a la diferencia que establece entre la vida premoderna y la vida moderna. La vida premoderna se corresponde con la vida en el rural o en la pequeña ciudad, cuyas dinámicas se contraponen a las de la gran urbe, anclada en el nacimiento de la modernidad.

Al contrastar ambas, Simmel aplica un paradigma evolucionista, influenciado por las teorías del darwinismo. En los semestres de invierno de 1886-1887 y 1888-1889 Simmel imparte dos seminarios en torno a las "consecuencias filosóficas del darwinismo" y en 1895 publica su ensayo Sobre la relación entre la doctrina de la selección y la epistemología. Hay que tener en cuenta además que su pensamiento está influenciado por el evolucionismo de Herbert Spencer, en particular el de sus First Principles, traducidos al alemán en 1875 , donde junto con su ley de la evolución expone el tránsito de la homogeneidad a la heterogeneidad. En la reflexión de Spencer en torno a la evolución de los organismos este proceso va asociado a un aumento de la complejidad y de la diferenciación (Frisby, 2008, p. 79, p. 150) ${ }^{6}$.

Ejemplo de homogeneidad sería la experiencia que ofrece una urbe histórica como Roma. En su lectura de Roma -una interpretación, como ha señalado Micaela Cuesta, más estética que sociológica (Cuesta, 2016, p. 25)- Simmel pone el acento sobre la unidad, la homogeneidad de estímulos que reúne:

"Del mismo modo que el misterioso encanto de las telas antiguas reside en que sus distintos olores, al compartir durante años un mismo destino, de sol y sombras, de humedad y sequedad, acaban conciliándose en una unidad que solo el tiempo da, así cabría decir que las cosas más alejadas y extrañas entre sí, por compartir la experiencia de estar en Roma y participar de su destino, experimentan una adaptación, un influjo recíproco, una integración por la que la significación individual de cada cosa alcanza su plenitud tanto como la alcanza la unidad en la que encajan como partes integrantes. Esa unidad produce un efecto psicológico, un entusiasmo, muy propio de Roma y que, de modo similar, solo se manifiesta ante los individuos más grandes." (Simmel, 2013, p. 16) ${ }^{7}$

Simmel aplica a Roma un análisis de ciudad intemporal-de ciudad eterna- que no se ve afectada por las transformaciones propias de la gran urbe y en la que los elementos que la integran están llamados a formar parte de ese todo unitario que resulta en la experiencia de la capital romana. De este modo, en Simmel parece confluir el análisis estético con la homogeneidad premoderna, frente a una lectura sociológica, la propia de la urbe moderna que, como veremos más adelante, es en esencia fragmentaria y cambiante:

"El que en Roma uno no se sienta apabullado sino, por el contrario, en plenitud de sí mismo es sin duda reflejo de que la autonomía de nuestro espíritu ha alcanzado su máxima expresión [...] Allí donde las impresiones y los entusiasmos surgen espontáneamente y no reclaman de nosotros un ejercicio que ponga en juego nuestras fuerzas para forjarnos una imagen de las cosas, el recuerdo será borroso y pasajero. Porque por profunda y conmovedora que haya sido la impresión, esta seguirá siendo en lo más íntimo de nuestra alma un elemento extraño destinado a desvanecerse sin dejar rastro. Solo cuando el alma pone en juego su propia interioridad y recubre con la trama de su propia actividad las impresiones recibidas desde fuera logra que estas sean suyas, logra apropiárselas. La conciencia superior, el reflejo de su libertad y de su dominio, es su capacidad para establecer vínculos entre cosas dispares apreciando al mismo tiempo la diversidad de las mismas, por cuanto unidad y diversidad se presuponen recíprocamente. En ningún otro lugar [como en Roma] un alma recibe tanto y debe acometer tanto para dar forma a la imagen. [...] Sin duda, la multiplicidad de efectos e interpretaciones que produce Roma se corresponde con el principio mismo de la vida, sobre el que se asienta, a mi entender, el carácter único de su estética." (Simmel, 2013, pp. 19-20).

Simmel deja entrever aquí cómo se produce la recepción de impresiones por parte del individuo en el caso de la ciudad de Roma, donde todas ellas presentan cierto carácter de unidad. El espíritu del urbanita ha de hacer un esfuerzo por aproximarse a la ciudad, lo cual permite interiorizarla, apropiársela. Frente al "apabullamiento" que el individuo sufre en la urbe moderna, Roma daría, para Simmel, la medida exacta del juego de intercambio entre los estímulos que la ciudad genera y la capacidad de recepción subjetiva de los mismos por parte del individuo.

La ciudad de Roma parece ubicarse en un punto intermedio entre la ciudad premoderna y la ciudad moderna. Así, la homogeneidad de estímulos sería característica de la vida urbana en la Antigüedad -en la antigua Grecia- y en la Edad Media, donde se "impuso al individuo una limitación tal de sus movimientos y de sus relaciones externas, de su autonomía y su diferenciación social, que harían la existencia asfixiante 
al hombre moderno. Aún hoy el habitante de la gran ciudad siente, trasplantado a la pequeña ciudad, una sensación de asfixia" (Simmel, 2016, p. 69). Esta opresión vendría de la imposibilidad de movimiento y del estatismo dominante que caracteriza la vida premoderna: la de los tiempos pasados y la de las comunidades de pequeñas dimensiones. Roma mantendría la homogeneidad de la ciudad premoderna pero con características de la gran urbe, lo cual favorecería una cierta libertad individual y flexibilizaría las relaciones.

Así, Simmel contrapone la falta de libertad individual a la heterogeneidad de la vida en la gran urbe propia de una economía capitalista donde se bombardea a sus habitantes con nuevos productos destinados "a consumidores que son completos desconocidos y que nunca han entrado en la esfera visual del productor propiamente dicho" (Simmel, 2016, p. 62). En este último caso, los estímulos urbanos -lo que Simmel enmarca dentro de la cultura objetiva- acaban imponiéndose sobre la cultura subjetiva del individuo, sobre su vida mental. La asimilación de este ritmo intenso de la vida moderna a través de los sentidos es objeto de reflexión para Simmel, es más, nuestro autor inserta la sensorialidad dentro de su paradigma evolucionista. Veamos de qué manera.

\section{LA PERCEPCIÓN SENSORIAL EN LA COTIDIANEIDAD URBANA}

Como anunciaba al inicio, Simmel presenta una lectura sociológica de la percepción sensorial en su "Digresión sobre la sociología de los sentidos" (1907), en la que, además, integra las facultades sensibles en un paradigma evolucionista. Y así, según Simmel, "a medida que se afina la civilización, decrece la agudeza perceptiva de los sentidos, al paso que aumenta la sensibilidad para lo agradable o desagradable de la impresión" (Simmel, 1977, pp. 687-688). Esta frase hay que entenderla, por un lado, en el marco del auge del evolucionismo en las décadas finales del siglo XIX $y$, por otro, como parte de toda una reflexión en torno al lugar que este momento bisagra entre los siglos XIX y XX ocupaba en la evolución de los sentidos, lo cual implica el enfrentamiento entre posturas contrapuestas. Si bien Simmel defiende una progresiva pérdida de refinamiento en la percepción sensorial, para otros intelectuales contemporáneos, como es el caso de Valle-Inclán, "hoy percibimos relaciones de colores y sonidos que hace años no se producían". No hay duda de que en el caso del escritor gallego su criterio viene definido por el contexto modernista en el que se encuentra inserto, del que es testimonio su afir- mación, perteneciente al mismo texto: "la condición característica de todo el arte moderno, y muy particularmente de la literatura, es una tendencia a refinar las sensaciones y acrecentarlas en el número y en la intensidad" (Hernández Barbosa, 2014, pp. 285-286). En todo caso, ambas posturas deben enmarcarse en los debates en torno a la sensorialidad que condujeron a enfrentar dos corrientes diferenciadas: aquella que ve el fin de siglo como un momento de esplendor en la evolución de la percepción sensorial, y la que, por el contrario, lo entiende como una época de degeneración de la especie ${ }^{8}$.

Para Simmel las nuevas condiciones que impone la urbe moderna suponen un cambio en el comportamiento de los sentidos. La socióloga Olga Sabido Ramos apunta a la categoría de "proximidad sensible" como central en la sociología simmeliana, referida a la dimensión interactiva de las disposiciones de los sentidos (Sabido Ramos, 2014, p. 85). El sentido de la vista sería el que mejor encarna esta reconfiguración: "Antes de que en el siglo XIX surgiesen los omnibús, ferrocarriles y tranvías, los hombres no se hallaban nunca en la situación de estar mirándose mutuamente, minutos y horas sin hablar" (Simmel, 1977, p. 681). El auge de los medios de transporte colectivos transforma las relaciones humanas situando frente a frente a individuos desconocidos.

El sentido de la vista establece una interacción poderosa e inmediata entre individuos a través de lo que Simmel denomina "el milagro de la mirada" (Simmel, 1977, p. 684), caracterizada por su reciprocidad y bidireccionalidad (Sabido Ramos, 2017, p. 384). Esta comunión entre personas que se genera instantáneamente proporcionaría conocimiento entre ellas simplemente a través de la contemplación del rostro, puesto que este es revelador de facetas de la persona intransmisibles de otro modo, de ahí su naturaleza enigmática, "por eso el que ve, sin oír, vive más confuso, desconcertado e intranquilo, que el que oye sin ver" (Simmel, 1977, p. 681). Simmel apunta que puesto que la vista constituye un medio de conocimiento por naturaleza más enigmático que el oído -en tanto el oído se asimila a la recepción a través del lenguaje hablado-, el ocularcentrismo que rige la vida en la metrópolis contribuye al desconcierto del ciudadano, de ahí el "carácter problemático que aqueja al sentimiento moderno de la vida" (Simmel, 1977, p. 681). Esto contrastaría con el predominio del lenguaje, y su recepción a través del oído, como medio fundamental de conocimiento del otro según había imperado hasta entonces, tanto a través del contenido del discurso 
como de las modulaciones a que está sujeto. Dentro de las especificidades que Simmel atribuye a cada uno de los sentidos en la sociedad contemporánea apunta que en un grado inferior respecto a la comunicación entre personas estaría el sentido del olfato, que para Simmel detenta una significación sociológica menor que la vista y el oído (Simmel, 1977, p. 686).

Por otra parte, para Simmel "solo cabe 'poseer' lo visible", mientras que 'lo puramente audible', desparece" (Simmel, 1977, p. 684). Como consecuencia de ello, el sentido de la vista quedaría asimilado a la dinámica propia del capitalismo en tanto materialidad visible que se puede poseer. En cambio, el oído no podría apropiarse del emisor sonoro: "lo que uno dice a otro, lo oirían incontables personas si estuviesen presentes", "el oír es, en su esencia, algo supraindividual; todo lo que suena en un espacio han de oírlo cuantos se hallan en él, y el hecho de que uno lo perciba, no priva de percibirlo a los demás" (Simmel, 1977, p. 684).

Estas premisas que Simmel asume como universales son, en realidad, culturales y, de hecho, en la propia época en que las expone, las condiciones de escucha estaban experimentando un cambio sustancial. Así, en 1907, cuando publica su texto sobre los sentidos, ya se había dado a conocer un dispositivo que permitía la comunicación a distancia: el teléfono, patentado en 1876 y que permite hacer de lo audible una realidad individual (y no colectiva). Es también el caso del théâtrophone, un servicio de emisión telefónico que posibilitaba, mediante subscripción, seguir en directo en diversos puntos de la ciudad de París diferentes performances teatrales y que estuvo vigente desde 1889 hasta mediados de la década de $1930^{10}$. Asimismo, lo audible podía llegar a constituirse en una realidad susceptible de ser poseída, tanto a través del elemento físico del disco como del propio aparato emisor del sonido. Es el caso del fonógrafo, comercializado a partir de su invención en 1877. Los cambios tecnológicos que lleva implícita la aparición de nuevos dispositivos vinculados a los sentidos ponen en cuestión algunos de los postulados que Simmel plantea en el artículo objeto de estudio quien, si bien se hace eco de ciertos cambios sociológicos que se estaban operando en el momento coetáneo, deja al margen de su reflexión toda una serie de avances tecnológicos encargados de conformar al espectador moderno que discuten sus reflexiones esencialistas en torno a los sentidos.

Si bien en su reflexión incorpora el papel de otros ámbitos sensoriales, Simmel entiende la sociabiliza- ción mediante el sentido de la vista como propia de la experiencia moderna:

"Las comunicaciones modernas hacen que la mayor parte de las relaciones sensibles entabladas entre los hombres queden confiadas, cada vez a mayor escala, exclusivamente al sentido de la vista". (Simmel, 1977, p. 681).

En efecto, la asimilación entre visualidad y cultura moderna centró los estudios de la modernidad, dando como resultado un ocularcentrismo que desde finales del siglo XX viene siendo revisado. La modernidad se reivindica actualmente como un cambio de paradigma que afectó no únicamente a la visualidad sino al conjunto de ámbitos sensoriales, con particular énfasis en lo aural y lo háptico, lo cual cuestiona una concepción del mundo moderno asociada exclusiva o preeminentemente a lo visual. Este nuevo paradigma se ha desarrollado en buena medida gracias a los estudios de "sensory history" (Boutin, 2015, p. $137)^{11}$. Incluso ciertos parámetros históricos confirman que ya en el fin de siglo la visión se entendía asociada a la corporalidad (Hernández Barbosa, 2017a, pp. 359-370). La mirada ha de ser estudiada en su integración con la corporeidad implícita en el acto de observar, hablaríamos en este caso de una "embodied vision". De este modo, la novedad que supone la mirada a los ojos entre dos ciudadanos en el autobús no puede aislarse del ambiente sonoro que en el interior del vehículo se genera -y que dificultaría la conversación- así como del roce de los cuerpos, inevitable en un espacio tan estrecho. Si la nueva urbe impone nuevas condiciones para la experiencia sensorial, la naturaleza de esta no puede abordar aisladamente cada uno de los sentidos ${ }^{12}$.

\section{La psicología del individuo ante la ciudad moderna: individualización intelectual y hastio}

Simmel planteaba cómo la ciudad moderna implica nuevas configuraciones espaciales que juegan con nociones de distancia y cercanía, estatismo y movilidad (Frisby, 2008, p. 50). Jonathan Crary ha puesto sobre la mesa cómo precisamente en este contexto al que Simmel se refiere -Crary concreta los años que median entre 1880-1905- se gestaron las nuevas condiciones de percepción propias de la modernidad. Entre ellas se encuentra esa "recepción en estado de distracción" a la que Simmel alude, recepción marcada por experiencias de fragmentación, shock y dispersión (Crary, 1999/2008, p. 11).

Estas experiencias se manifestarían tanto en las relaciones interpersonales como en las que la urbe genera 
en el individuo. En el caso de las primeras, el aumento de contactos inesperados, así como la fugacidad de los mismos, implicaría para Simmel la ruptura del equilibrio cotidiano tal y como imperaba hasta entonces. A su vez la urbe conlleva, según Simmel, una "sucesión rápida e ininterrumpida" de estímulos -de imágenes, de informaciones- que el individuo recibe como impresiones. La lógica capitalista impone esta saturación de estímulos, aquella "en la que el que oferta ha de crear necesidades nuevas y cada vez más originales en el consumidor, al que no deja de cortejar. La necesidad de especializar los servicios que se prestan para encontrar una fuente de ingresos aún no agotada, una función que no sea fácilmente sustituida incita a la diferenciación, al refinamiento, al enriquecimiento de las necesidades del público" (Simmel, 2016, p. 73). Dentro de esta lógica capitalista no hay que olvidar el impacto de la industria publicitaria, presente tanto físicamente en la ciudad, por ejemplo, a través del cartelismo, como en los medios de comunicación.

Una muestra de la saturación de estímulos propia de este momento se produce en las exposiciones universales, que reúnen toda la producción cultural de una época en unos metros cuadrados de ciudad. Ya la prensa coetánea aludía a la concentración de estímulos que en ellas tenía lugar, por ejemplo a propósito de las atracciones que se presentan en la Exposición Universal de 1900: "Desde el punto de vista de la instrucción que nos hemos prometido, [...] estos inmensos panoramas se han vuelto demasiado vastos, contienen demasiadas cosas y, a menos que uno disponga de todo el tiempo del mundo, de piernas y ojos infatigables, conocimientos enciclopédicos, una memoria sin fallas, o que nos limitemos estrictamente a uno o dos aspectos concretos, se ha vuelto casi imposible recorrerlos de manera satisfactoria" 13 .

$\mathrm{El}$ individuo, en su intento por aprehender estas impresiones, por aislar unas de otras, consume según Simmel gran cantidad de energía mental. La urbe implica una cierta deficiencia de puntos de referencia en relación con la pequeña ciudad o con la vida rural; por ello el urbanita gasta más energía ya que el ritmo dominante en estas últimas es "más lento, habitual y regular", la vida es más predecible. Esto supone la "intensificación de la vida nerviosa", que a su vez exige una mayor intelectualización. La actividad mental es demandada como producto de la capacidad del ser humano para adaptarse, para reaccionar ante nuevos estímulos, lo que nos habla de la aplicación por parte de Simmel, una vez más, de las teorías darwinistas al medio urbano ${ }^{14}$.
Esta exigencia adaptiva explicaría el "carácter intelectual de la vida anímica de la gran ciudad", frente a la vida en la pequeña ciudad, que "descansa en los lazos afectivos y en las relaciones emocionales" (Simmel, 2016 , p. 60). Simmel privilegia el carácter intelectual de la vida en la gran ciudad, donde no sería posible esa reacción interna y emocional constante hacia cada persona: "Si el contacto externo incesante con innumerables personas tuviese que encontrar respuesta en una cantidad igual de reacciones internas, como ocurre en la pequeña ciudad, en la cual casi todos se conocen y mantienen una relación positiva, uno terminaría por atomizarse interiormente del todo y caería en un estado psicológico inimaginable" (Simmel, 2016, p. 67). Por otra parte, dentro de su paradigma evolutivo, Simmel entiende que "el sentir, en comparación con el pensar, es sin duda, filogenéticamente, el estado inferior" (Frisby, 2008, p. 170). Esta "cualidad racional" actúa como "protectora de la vida subjetiva contra la violencia ejercida por la gran ciudad" (Simmel, 2016, p. 61). Se produce así la paradoja de situar la cercanía física ante la lejanía intelectual, lo que explicaría que "el hecho de que en ninguna otra parte uno se sienta más solo y abandonado que entre el gentío de la gran ciudad no sea [es] más que el reverso de esta libertad" (Simmel, 2016, p. 71). El vínculo entre los sentidos y la ciudad debe entenderse en Simmel como parte de su conceptualización de la dialéctica entre cultura subjetiva y cultura objetiva, según la cual la cultura objetiva se interpone a la existencia humana y se acaba incorporando a la cultura subjetiva (Frisby, 2008, pp. 35-43).

De este modo, en la gran urbe se crean las condiciones para la individualización, del "ser para sí mismo" (Zabludovsky y Sabido, 2014, p. 20), del que se exige una reacción ante las formas de interacción y los estímulos impuestos. Así, nos dice que "los problemas más profundos de la vida moderna se derivan de la aspiración del individuo a preservar la autonomía e individualidad de su existencia frente a fuerzas sociales abrumadoras" (Frisby, 2008, p. 248), lo cual supone un caldo de cultivo para que la "personalidad se encargue crecientemente de sí misma", el surgimiento del sentimiento del yo por oposición al grupo, que evitaría pasar desapercibido en la multitud (Zabludovsky y Sabido, 2014, p. 52, p. 74). Su materialización a través de la vestimenta o del comportamiento en sociedad resultaría en las figuras del dandi y el esteta, representativas de una sensibilidad ubicada en los límites del sistema ${ }^{15}$. De hecho, en el Berlín de Simmel surgió un grupo contracultural denominado "Bohemios Berlineses" cuya vestimenta y estilo de vida se aproximaron a las del dandi (Sabido Ramos, 2017, p. 382). 
Una de las consecuencias del crecimiento de la intelectualización es el exceso de demanda del cerebro, con la aparición de nuevas patologías. Es el caso de la fatiga mental y, como derivación de esta, la neurastenia, que permitió otorgar entidad clínica a la fatiga patológica. La fatiga mental y su extensión en la neurastenia -literalmente "debilidad nerviosa"- eran consideradas signo de una sensibilidad excesiva producto de un agotamiento nervioso. El diagnóstico de esta afección experimenta gran auge en el mundo occidental a partir de 1880 , en particular gracias a los trabajos del neurólogo norteamericano George Miller Beard (1838-1883). La neurastenia se convirtió así en una de las neurosis asociadas a la nueva vida moderna, que podía vincularse tanto al exceso de estímulos sensibles como a la sobrecarga de trabajo mental (Hernández Barbosa, 2017b, p. 13). La acepción de Simmel encajaría en una concepción de la enfermedad que fusiona ambas causas.

Junto con la reacción mediante el intelecto, Simmel identifica otro tipo de respuesta ante el embotamiento de los estímulos: el hastío, la indiferencia, la desidia, que experimentan aquellos individuos que tienen dificultades a la hora de reaccionar ante la multitud de estímulos recibidos:

\footnotetext{
"Tal vez no haya un fenómeno anímico tan exclusivo de la gran ciudad como el hastío. Es en principio la consecuencia de aquellos estímulos nerviosos de rápida alternancia y acusada contradicción, de los que nos parecía surgir también la creciente intensidad intelectual de la gran ciudad; esto explica que personas de mente débil no suelen mostrarse hastiadas. Así como una vida de placeres inmoderados termina por causar hastío, ya que estimula tanto tiempo el sistema nervioso hasta alcanzar sus reacciones máximas que termina por no producir reacción alguna; del mismo modo, estímulos más inofensivos, por la rapidez y naturaleza contradictoria de sus variaciones, fuerzan respuestas tan violentas, sacuden el sistema nervioso con tal brutalidad que este agota sus últimas reservas energéticas y, al permanecer en el mismo medio social, no tiene tiempo de regenerarse. Esta incapacidad así originada de reaccionar a nuevos estímulos con la energía adecuada a ellos constituye ese hastío" (Simmel, 2016, p. 65).
}

El tedio -l'ennui- constituye otra de las grandes cuestiones del XIX, que empezó a considerarse mal epidérmico a partir de los años cuarenta. En el cambio de siglo, en 1903, el teórico francés Émile Tardieu le otorga entidad psicológica con tintes existencialistas (Tardieu, 1903/1913). Simmel, en esta misma línea, identifica el tedio como una afección específicamente urbana causada por una reacción de desidia mental ante la sobrestimulación.

Una de las grandes cuestiones que preocuparon a médicos e intelectuales del fin de siglo ante el efecto de la sobrestimulación es la atención, o más bien su ausencia. La inatención ante el exceso de estímulos empezó a verse como un problema en el marco de los métodos modernos de trabajo mecanizado, los cuales, a su vez, se encontraban en su origen. En el ámbito de la psicología experimental, figuras como Wilhelm Wundt o Alfred Binet, en dos contextos diferentes, se preocuparon por esta cuestión. Desde una lectura sociológica, Simmel se encuentra en un paradigma de análisis en el que la atención aparece como una actividad neutral, atemporal, que en el siglo XIX se ve distorsionada; es entonces cuando aparece el "problema de la atención". La lectura de Simmel, que entiende la percepción distraída como una característica fundamental para entender la subjetividad moderna, ha sido revisada por Crary, quien ha puesto sobre la mesa cómo la cuestión de la atención nos habla de un modelo específico de conducta dentro de una estructura histórica marcada por las condiciones propias de la modernidad mecanizada. La lógica capitalista exige desplazar la atención de una cosa a otra rápidamente, haciendo de la movilidad, la novedad y la distracción elementos fundamentales en la experiencia perceptiva. El que la distracción aparezca como problema a finales del siglo XIX no puede desligarse de la construcción paralela de un observador atento a múltiples campos (Crary, 2008, p. 23, pp. 3738 , pp. 55-57). Las novedades tecnológicas requieren del espectador un esfuerzo que, como hemos visto en el caso del público asistente a exposiciones universales, este logra identificar en ocasiones como exigencia difícilmente asumible.

Los textos abordados permiten poner en relación la reflexión de Simmel con diferentes dimensiones de la cultura contemporánea: la evolución de la ciudad y su espectacularización, el auge del capitalismo, la aparición de nuevas enfermedades y de una nueva cultura material tecnológica. La visión de Simmel nos interesa por su identificación en el contexto de la metrópolis coetánea de nuevas condiciones sociales que afectan a la psicología del individuo. Estas circunstancias tienen que ver con el auge de los medios de transporte colectivos, el bombardeo de estímulos, los nuevos modos de relaciones personales, la libertad individual como reverso al incremento de la ansiedad o las nuevas problemáticas ligadas a la atención, todas ellas 
constitutivas del individuo moderno. Simmel posiciona de forma pionera la sensibilidad en el centro de la vida urbana, abordando los elementos definitorios de las nuevas relaciones que en su seno se generan ligadas, en gran medida, a la interacción entre individuos desconocidos. Esto supone una reconfiguración de la sensibilidad que otorga al sentido de la vista un lugar prioritario en el contacto entre extraños. En su aplicación del darwinismo al medio urbano, la adaptación que exigirían las nuevas condiciones de la ciudad moderna supone la aparición de afecciones específicas: el tedio y la neurastenia, dos reacciones opuestas ante los excesos de la sobrestimulación.

Deja, en cambio, al margen uno de los componentes rectores de la vida moderna: las nuevas tecnologías, cuyo abordaje hubiera conducido a Simmel por caminos, sin duda, diferentes. Habrá que esperar a la historia cultural y a los estudios visuales, casi un siglo después, para que este cruce protagonice la comprensión de la cultura moderna y permita entender como sociales-culturales presupuestos que Simmel aborda aquí como universales. Como es lógico, la lectura de Simmel está marcada por marcos de pensamiento propios de su época, es el caso de su interpretación de ciertas propiedades específicas que de manera esencialista aplica a los sentidos o el paradigma evolucionista en que inserta la psicología humana.

Resulta, sin embargo, difícil abstraerse de una lectura actualizada de los planteamientos de Simmel, en particular como definitorios de nuestra sociedad del capitalismo avanzado en que las grandes urbes rigen el mundo; en una configuración cada vez más protagonizada por el empeño en posicionarse ante la uniformidad propia de la globalización; en un mundo dominado por las comunicaciones -el marketing a distancia- más que nunca dirigido "a consumidores que son completos desconocidos y que nunca han entrado en la esfera visual del productor propiamente dicho", como señalaba Simmel para su propia época (Simmel, 2016, p. 62). En la misma línea de Simmel, un célebre pensador del fin de siglo, Max Nordau, autor del bestseller más controvertido de la década de 1890 (Maik, 1989, p. 607), predecía que "el final del siglo XX albergará a una generación a la que no le molestará leer una docena de metros cuadrados de periódicos al día, que le llamen por teléfono constantemente, pensar en los cinco continentes del planeta de forma simultánea, pasar la mitad de sus vidas en vagones de trenes o máquinas de volar [...] y que sabrá cómo encontrar la tranquilidad en medio de una ciudad poblada por millones de personas" (Crary, 1999/2008, p. 39). Al margen de los augurios apocalípticos de Nordau, cómo no reconocer nuestro mundo contemporáneo en su descripción.

\section{AGRADECIMIENTOS}

Este texto se enmarca dentro de los proyectos de investigación del Ministerio de Economía y Competitividad de España: Entre ciudades: paisajes culturales, escenas e identidades (1888-1929) (HAR2016-78745-P), Universitat de Barcelona, y Nuevos modelos de gobierno de las ciudades, e intervención en los espacios urbanos en el período postcrisis (CSO2016-75236-C21-R), Universidade de Santiago de Compostela.

\section{NOTAS}

1. João Silva apunta por ejemplo al caso de Lisboa y cita el de Río de Janeiro durante la primera década del siglo XX (Silva, 2017).

2. Asimismo, las transformaciones en cuanto al paisaje sonoro tienen que ver con el aumento de la velocidad de los vehículos y el intento de evitar los accidentes derivados de ello a través de los cláxones (Corbin, 2016, p. 54).

3. En el contexto berlinés al que Simmel estuvo vinculado habría que citar, en concreto, la Exposición Comercial de Berlín de 1896.

4. Este texto ha sido señalado por su carácter pionero en el ámbito de la sociología del cuerpo por autores como Bryan Turner, Deena Weinstein y Michel
Weinstein, Anthony Synnott, David Le Breton, Janet Stewart, Kelvin Low, Philip Vannini et al. (Sabido Ramos, 2017, p. 375, pp. 388-389)

5. Olga Sabido apunta como aportaciones destacadas de Simmel precisamente su importante papel en la definición de la modernidad y el "refinamiento de la mirada sociológica" (Sabido Ramos, 2014, p. 75).

6. En esta misma línea formula sus teorías el naturalista británico Ray Lankester, para quien la evolución de los organismos marcha en paralelo a la especialización y diferenciación de sus elementos. Como parte de esta teoría, el fenómeno de la sinestesia, que despertó el interés de la medicina en el fin de siglo, sería síntoma de evolución anómala, pues en vez de tender a la especialización lo haría hacia la reagrupación de las capacidades sensoriales (Hernández Barbosa, 2013, p. 127).

7. Este artículo sobre Roma fue originalmente publicado como "Rom. Eine ästhetische Analyse" en la revista semanal Die Zeit, Wiener Wochenschrift für Politik, Volkswirtschaft, Wissenschaft und Kunst, Viena, 28 de mayo de 1898.

8. Sobre esta cuestión presenté el paper: "Diversification or sensory unification? Ideas around the evolution of the senses in fin de siècle culture" en el congreso Medicine and Modernity, St Anne's College, Oxford, 10-11 de septiembre de 2016. 
9. Esta división de los sentidos entre superiores e inferiores estaba presente en la época desde el punto de vista ya no sociológico sino fisiológico. Es el caso de su consideración por parte de dos psicólogos españoles insertos en la tradición del krausismo. Por un lado, Giner de los Ríos denominaba a los sentidos de la vista y el oído los "sentidos zoológicos y teóricos por excelencia" ya que son los órganos receptivos más importantes en el acto del pensamiento. González Serrano, por su parte, se refiere a los sentidos de la vista y el oído como aquellos "principalmente instructivos"; en cambio el gusto y el olfato, "adheridos a la función nutritiva", serían sobre todo afectivos, teniendo en cuenta que "cuanto más nos afecta y emociona un sentido menos nos instruye" (Hernández Barbosa, 2014, pp. 294-295).

10. El théâtrophone se presentó en la Exposición Universal de 1889 (Drie, 2015, p. 75).

11. Los planteamientos de la "sensory history" suponen una revisión in nuce de los postulados que privilegian la visuali-

\section{BIBLIOGRAFÍA}

Baudelaire, Ch. (1863/2000). El pintor de la vida moderna. En Prado, J. del y Millán Alba, J. A. (eds. y trads.). Obras completas. Madrid: Espasa Calpe, pp. 1369-1417.

Bertrand, A. (1996). París y la electrificación de Francia. Revista de Historia Industrial, 9, pp. 39-52.

Boutin. A. (2015). City of Noise. Sound and Nineteenth-Century Paris. Champaign: University of Illinois Press. https://doi.org/10.5406/illinois/9780252039218.001.0001

Corbin. A. (2016). Urban Sensations: The Shifting Sensescape of the City. En: Classen, C. (ed.). Cultural History of the Senses in the Age of Empire. London: Bloomsbury, pp. 47-67.

Crary, J. (1999/2008). Suspensiones de la percepción. Atención, espectáculo y cultura moderna. Madrid: Akal.

Cuesta, M. (2016). Estudio introductorio. En: Simmel, G. Las grandes ciudades y la vida intelectual. Madrid: Hermida editores, pp. 11-55.

Drie, M. van. (2015). Hearing through the théâtrophone: sonically constructed spaces and embodied listening in late dad. Entre sus representantes citaré los siguientes nombres, sin pretensiones de exhaustividad: Constance Classen, Mark M. Smith, David Howes y Robert Jütte, así como la labor pionera de Alain Corbain, como parte de una historia de las mentalidades.

12. Olga Sabido Ramos matiza algunos de los postulados presentados en la "Digresión sobre la sociología de los sentidos" a partir de otros escritos de Simmel. Por ejemplo, apunta a la lectura multisensorial que hace Simmel de la música en: Simmel, G. (1882/2003). Estudios psicológicos y etnológicos sobre música. Buenos Aires: Gorla. (Véase Sabido Ramos, 2017, p. 387).

13. "Au point de vue de l'instruction que l'on s'en promet, enfin, ces immenses panoramas sont devenus trop vastes, contiennent trop de choses, et, à moins de posséder, avec l'entière liberté de son temps, des jambes et des yeux infatigables, des connaissances encyclopédiques, une mémoire sans défaillance, ou de se renfermer rigoureusement dans le cercle d'une ou de deux spécialités, il est devenu pres-

19th century French theatre. Sound Effects-An Interdisciplinary Journal of Sound and Sound Experience, 5 (1), pp. 74-90. https://doi.org/10.7146/se.v5i1.23310

Faria Peres, F. de, Flores Durán, P. R., Pacini de Medeiros y Alburquerque, N. (2011). A 'sensibilidade' de Simmel: notas e contribuições ao estudo das emoções. Revista Brasileira de Sociologia da Emoção, 10 (28), pp. 93-120.

Frisby, D. (2008). Georg Simmel. México: Fondo de Cultura Económica.

García Alonso, R. (1995). Ensayos sobre literatura filosófica. G. Simmel, R. Musil, R. M. Rilke, K. Krauss, W. Benjamin y J. Roth. Madrid: Siglo Veintiuno de España Editores.

Gluck, M. (2008). Popular Bohemia. Modernism and Urban Culture in Nineteenth-Century Paris. Cambridge, MA: Harvard University Press.

Hahn, H. H. (2009). Scenes of Parisian Modernity: Culture and Consumption in the Nineteenth Century. New York: Palgrave Macmillan. https://doi. org/10.1057/9780230101937

Hernández Barbosa, S. (2013). Sinestesias. Arte, literatura y música en el París fin de siglo, 1880-1900. Madrid: Abada. que impossible de les parcourir avec un profil réel et durable" (Passy, 1899, p. 3). Traducción de la autora.

14. Una de las aplicaciones más célebres de las teorías darwinistas al ámbito social había sido la planteada por Hippolyte Taine en 1881, al integrar los conceptos de selección natural y adaptación al medio en su explicación de la producción artística de una época (Taine, 1881/1968).

15. La figura del dandi se ha interpretado históricamente como opuesta a la sociedad burguesa emergente. Un ejemplo sería el protagonista de À Rebours (1884), la novela de Huysmans, que rechaza toda forma de materialismo. Desde los cultural studies, sin embargo, se han planteado lecturas que permitirían integrarlo en la cultura de masas (Gluck, 2008). Por otra parte, a partir de una lectura del actor social simmeliano, Daniel Hurtado Cano establece tres tipologías de habitantes de la urbe moderna: el actor (de teatro), el aventurero y quien está siempre sujeto a la moda (Hurtado, 2013).
Hernández Barbosa, S. (2014). Discursos sobre la sensorialidad en el fin de siglo español: del krausismo a la ciencia experimental. Revista de Estudios Hispánicos, 48 (2), pp. 285-305. https://doi. org/10.1353/rvs.2014.0039

Hernández Barbosa, S. (2017a). Beyond the visual: panoramatic attractions in the 1900 World's Fair. Visual Studies, 32 (4), pp. 359-370. https://doi.org/10.10 80/1472586X.2017.1288071

Hernández Barbosa, S. (2017b). Los discursos en torno a la sensorialidad en el fin de siglo: Luis Simarro y su lectura holística del ser humano, entre lo individual y lo colectivo. Bulletin of Spanish Studies, 94 (7), pp. 1127-1144. https://doi.org/1 $0.1080 / 14753820.2017 .1317085$

Hurtado Cano, D. (2013). Georg Simmel: el laberinto urbano y la gradación infinita de la vida. Revista Colombiana de Sociología, 36 (2), pp. 81-92.

Maik, L. L. (1989). Nordau's Degeneration: The American Controversy. Journal of the History of Ideas, 50 (4), pp. 607-623. https://doi.org/10.2307/2709800

Marrey, B. (1979). Les Grands Magaisns des origins à 1939. Paris: Picard. 
Passy, F. (1899). L’Exposition de 1900. La Grande Revue de l'Exposition. Suplemento ilustrado de la Revue des Revues, 1, pp. 1-5.

Sabido Ramos, O. (2014). De la sensibilidad simmeliana a la sensibilidad como objeto de investigación. Hacia una reflexión sociológica de la sociología. En: Suárez, H. J. y Pirker, K. (coords.). Sociólogos y su sociología. Experiencias en el ejercicio del oficio en México. México D. F.: Universidad Nacional Autónoma de México, pp. 71-92.

Sabido Ramos, O. (2017). Georg Simmel y los sentidos: una sociología relacional de la percepción. Revista Mexicana de Sociología, 79 (2), pp. 373-400.
Silva, J. (2017). Porosity and Modernity Lisbon's Auditory Landscape from 1864 to 1908. En Biddle, I. y Gibson, K. (eds.). Cultural Histories of Noise, Sound and Listening in Europe, 1300-1918. Abingdon: Routledge.

Simmel, G. (1908). Sociology of the Senses: Visual Interaction. Leipzig: Dunker und Humblot, pp. 356-361.

Simmel, G. (1977). Digresión sobre la sociología de los sentidos. Sociología. Estudios sobre las formas de socialización. Madrid: Revista de Occidente, pp. 676695.

Simmel, G. (2013). Roma, Florencia, Venecia. Madrid: Casimiro.
Simmel, G. (2016). Las grandes ciudades y la vida intelectual. Madrid: Hermida editores.

Taine, H. (1881/1968). Filosofía del arte. Madrid: Espasa Calpe.

Tardieu, E. (1903/1913). L'Ennui. Étude phsychologique. Paris: Librairie Félix Alcan.

Zabludovsky, G. y Sabido, O. (2014). Estudio introductorio. En Simmel, G. Sociología: estudios sobre las formas de socialización. México: Fondo de Cultura Económica. 